\title{
Label free sensing with Terahertz multiple ring resonators
}

\author{
Xaing Bai, Lujun Zhe* \\ School of Electrical and Computer Engineering, Xiangtan University \\ * Corresponding author: lujun.zhe67@gmail.com
}

\begin{abstract}
Apart from their relevance for spectroscopy and imaging, terahertz signals have attracted a lot of interest for sensing. In this paper, a label free terahertz sensor is proposed, which can be employed to detect the presence of molecules in the environment. The proposed sensor consists of an array of ring resonators, resonating at the frequency of $f=1.2 \mathrm{THz}$. By providing full-wave numerical simulations, it is shown that the proposed sensor is able to sense the variation of the refractive index in the environment. The proposed structure is found to show larger sensitivity compared to previous reports. Our findings provide a novel platform to realize label free terahertz sensors with extremely large sensitivity.
\end{abstract}

Index Terms - Terahertz sensing, Graphene, Surface plasmon polaritons, Metamaterials, Ring resonators, Terahertz spectroscopy.

\section{INTRODUCTION}

$\mathrm{THz}$ band radiation is a part of the electromagnetic spectrum, occupying the frequency between $f=0.1-$ $10 \mathrm{THz}$ [1-10]. The waves in this frequency have some common properties. For instance, they can penetrate into various kinds of dielectric materials. Due to the lack of efficient sources and detectors, this frequency spectrum is called the THz gap. Remarkably, the energy of photons in the $\mathrm{THz}$ is smaller than the bandgap of the dielectric materials. Therefore, $\mathrm{THz}$ waves can penetrate in these materials. The transmitted wave can be employed to characterize the properties of materials, allowing one nondestructive evaluation, security and biomedical sensing [11-20].

One important application of terahertz waves is label-free sensing [], which can be used for molecular sensing, for instance. The usage of label-free technique prevents the challenges associated with labeling, including preparation of the sample. One of the most important way for label free sensing is based on refractive index changes. In this method, the structure senses biomolecules presence directly from the variation of the refractive index of the cladding medium. Many previous reports exist on the optical structures to achieve label-free sensing. One of the most important classes of such sensors are surface plasmon resonance (SPR) based sensors [21-25].

The working principle of the SPP sensors are the following: since the power of surface plasmon modes is mainly confined in an interface between metal and dielectric, it is sensitive to the changes in the refractive index of cladding material.
The oscillation of the electrons of a material on the edge of an interface made of a dielectric and metal is the basic principle of surface plasmon polaritons at any frequency range. The propagation loss of the surface plasmon polaritons in the spectrum is high. However, their localization is appropriate for sensing the changes in the dielectric refractive index. While various sensors are being created these days, the $\mathrm{THz}$ frequency with its exotic features is of utmost interest for biosensor researchers. One important property is that the associated phononic modes of many macro molecules like DNAs are in this particular range. This makes terahertz sensors perfect candidates to detect chemical matters more efficiently [8]. The absorption loss of $\mathrm{THz}$ SPPs on the interface of the two media is much smaller compared to the visible surface plasmon polaritons. Yet, they have very small localization to a dielectric material. As a result, surface plasmon polaritons are not appropriate for integration. This issue can be addressed in various approaches. Replacing metals with graphene strips can be a solution to solve this problem.

Graphene [26-36] is a thin layer carbon atom. The atoms of the monolayer are tightly packed into a two-dimensional hexagonal lattice of atoms. In particular, graphene can be considered as the two dimensional version of the bulky graphite. Research has been demonstrated that, in $\mathrm{THz}$ frequency regime, the surface conductivity of graphene flakes become imaginary, a property which allows the graphene strip to exhibit TM plasmonic modes. These modes have a much smaller effective wavelength than air, enabling miniaturization. Importantly, the graphene surface conductivity can be tuned dynamically over a wide range of frequencies, by just adding a bias or chemical doping. As a result, the propagation constant corresponding to the plasmonic mode can be dynamically adjusted. This enables reconfiguration of the associated device [37-46]. Moreover, confining electromagnetic fields in a short volume than regular materials has made graphene as a perfect choice for realization of nano structures.

In this paper, it is proposed to perform label free sensing by means of an array of surface plasmon polariton ring resonators. The ring resonators of the array are made of graphene strips sandwiched between two dielectric layers with different refractive indexes. It is shown that the proposed structure is capable of sensing the changes in the refractive index of the environment through a shift in the resonance frequency of the ring resonator. 


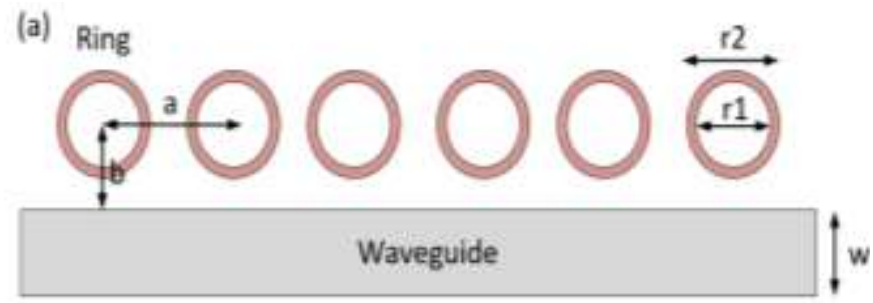

(b)

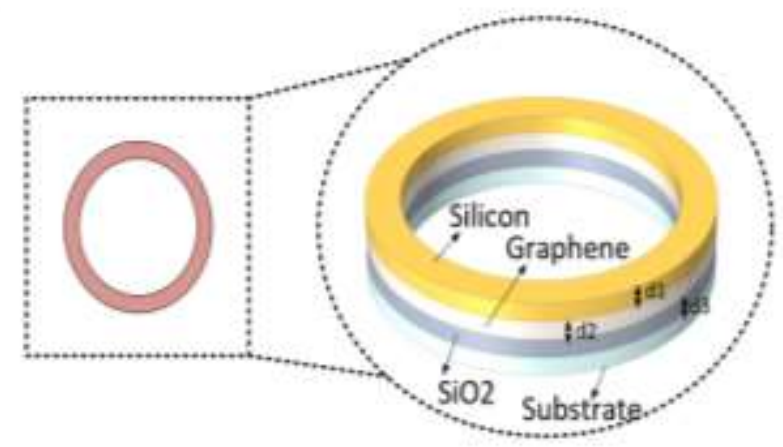

Fig. 1: a) Proposed structure for performing $\mathrm{THz}$ label free sensing. The structure is composed of an array of ring resonators. $\mathrm{B}$, structure of the ring resonators of the array

By providing the results of our finite element time domain simulations, it is shown that the proposed structure show a larger sensitivity compared to previous reports. It is further investigated that by tuning the chemical potentials of the graphene strips, one can reconfigure the proposed sensor to different frequencies. The chemical potentials of the graphene strips can be easily reconfigured using a change in the voltage applied to it. Alternatively, it is possible to change the chemical potentials of the graphene strips using chemical doping. We further investigate the effect of this change in the chemical potentials on the sensitivity and figure of merit of the proposed sensor. The proposed results provides a new route for designing ultra-sensitive label free Terahertz sensing devices, whose sensitivity and other associated parameters may be tuned in a dynamic way.

\section{PROPOSED STRUCTURE}

The proposed structure is shown in Fig.1. As it is observed, the proposed structure includes an array of split ring resonators. An optical waveguide excites the structure. Each ring resonator consists of a graphene strip, sandwiched between two dielectrics, namely $\mathrm{Si}$ and $\mathrm{SiO}_{2}$. The entire structure is placed on top of a silicon substrate.

In order to characterize the structure, we use Lumerical software, a full-wave electromagnetic solver based on finite domain time difference method (FDTD). The graphene strips in our structure is modeled based on an impedance layer. The surface conductivity of this impedance layer is defined by the conductance of the sheet, determined by the so-called Kubo formula.
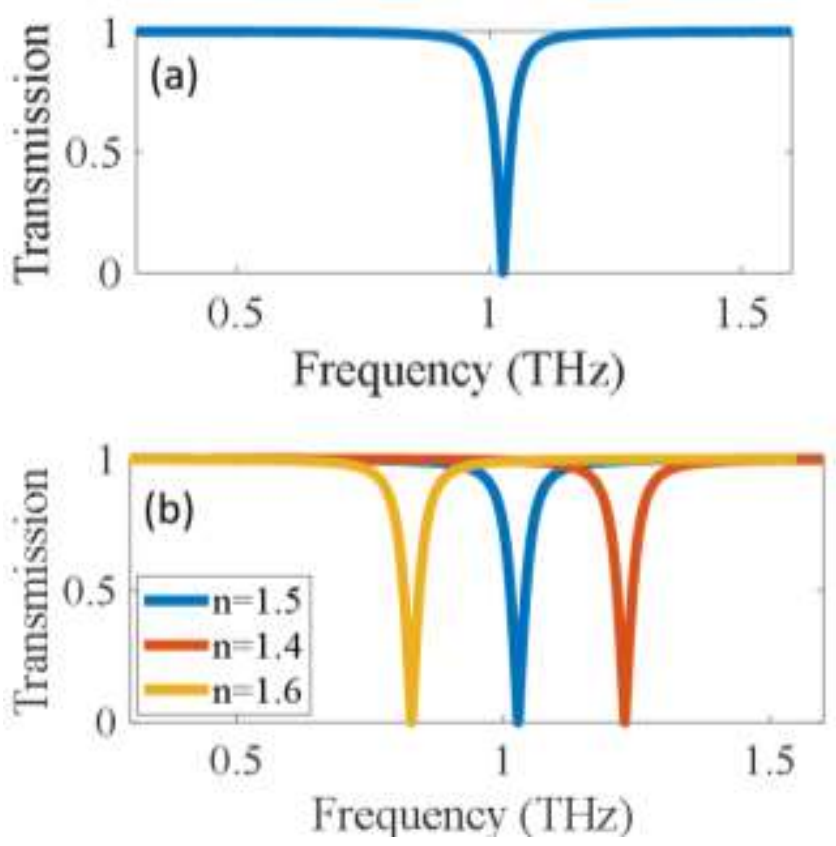

Fig. 2: a) Transmission spectrum of the proposed structure (b) variation of the transmission spectrum as a function of the refractive index of the top layer

The dielectrics of the structure are modeled using a blank material whose refractive index is in accordance with the permittivity of the material. The corresponding transmission coefficient of the structure is numerically calculated and shown in Fig. 2. As observed, the obtained result shows a resonance at the resonance frequency of the structure.

The obtained resonance is then used to perform label free sensing as it is described in what follows. Suppose that the presence of the molecules in the environment causes a change in the refractive index of the environment. This change can create a shift in the spectrum of the system. This effect is demonstrated in Fig. 3 of the manuscript, where the variation of the transmission spectrum of the system as a function of the refractive index of the cladding, i.e. the environment surrounding the structure is reported. As it is observed, the change in the refractive index has led to a shift in the spectrum. This shift can be used to perform label free sensing. In order to unambiguously investigate the sensitivity of the sensor under study, the variation of the resonance frequency shift as a function of the change in the refractive index is calculated and reported in Fig. 4. The figure reports this variation for various values of chemical potentials. The slope of these curves determines the sensitivity of sensor for that particular chemical potential.

To assess the functionality of the sensor, the quality factor of the sensor is another important factor that must be considered. The parameter $\mathrm{Q}$ can be obtained making use of $3 \mathrm{~dB}$ bandwidth. As it is known, one common technique to obtain the quality factor of a resonator is based on using the complex resonance frequency. The quality factor of the obtained resonators were achieved to be $Q=1895$. 
bioRxiv preprint doi: https://doi.org/10.1101/2021.03.07.434261; this version posted March 8, 2021. The copyright holder for this preprint (which was not certified by peer review) is the author/funder. All rights reserved. No reuse allowed without permission.

$>$ REPLACE THIS LINE WITH YOUR PAPER IDENTIFICATION NUMBER (DOUBLE-CLICK HERE TO EDIT) <

Note that, in general, there exist a tradeoff between the sensitivity and quality factor of the proposed sensor. In particular, one requires to determine if the high $Q$ is the important factor or a higher sensitivity matters. The Figure of Merit (FOM) is a parameter that can be considered as a perfect factor which takes into account both of these parameters. The FOM of the sensor under study is calculated to be $\mathrm{FOM}=96$.

To conclude, in this paper, we discussed designing a label free $\mathrm{THz}$ sensor. The proposed sensor was based on an array of graphene ring. By providing the results of our full-wave

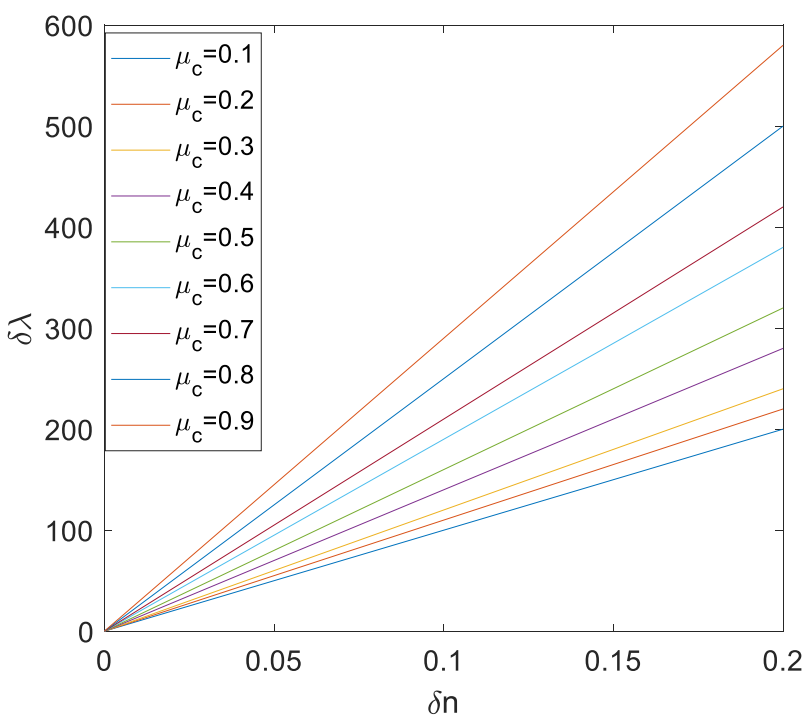

Fig. 3: Sensitivity of the proposed sensor as a function of the Fermi level of graphene layers. The sensitivity is determined by the slope of the curves

numerical simulations, we showed that the proposed sensor is capable of sensing the changes in environment through a shift in the spectrum of the system. The designed system is a appealing example of a terahertz bio sensor that provides a large sensitivity, quality factor as well as figure of merit. The generalization of the approach to the case of ring resonators supporting Fano or Kerkerr resonances can be a trivial extension of this research work [47-49].

\section{REFERENCES}

[1] Siegel, Peter H. "Terahertz technology." IEEE Transactions onmicrowave theory and techniques 50.3 (2002): 910-928.

[2] Ferguson, Bradley, and Xi-Cheng Zhang. "Materials for terahertzscience and technology." Nature materials 1.1 (2002): 26-33.

[3] Tonouchi, Masayoshi. "Cutting-edge terahertz technology." Nature photonics 1.2 (2007): 97-105.

[4] Lee, Y. S. (2009). Principles of terahertz science and technology (Vol. 170). Springer Science \& Business Media.

[5] Zangeneh-Nejad, Farzad, and Reza Safian. "Graphene-based archimedean spiral THz antenna." 2014 Third Conference on Millimeter-Wave and Terahertz Technologies (MMWATT). IEEE, 2014.

[6] Hu, Binbin B., and Martin C. Nuss. "Imaging with terahertz waves." Optics letters 20.16 (1995): 1716-1718.

[7] Fattinger, Ch, and D. Grischkowsky. "Terahertz beams." Applied Physics Letters 54.6 (1989): 490-492.

[8] Chan, W. L., Deibel, J., \& Mittleman, D. M. (2007). Imaging with terahertz radiation. Reports on progress in physics, 70(8), 1325.
[9] Zangeneh-Nejad, Farzad, Navid Barani, and Reza Safian. "Temperature dependance of elctromagnetic radiation from terahertz photoconductive antennas." Microwave and Optical Technology Letters 57.11 (2015): 2475-2479.

[10] Beard, Matthew C., Gordon M. Turner, and Charles A. Schmuttenmaer. "Terahertz spectroscopy." (2002): 7146-7159.

[11] Mittleman, D. M., et al. "Recent advances in terahertz imaging." Applied Physics B 68.6 (1999): 1085-1094.

[12] Chen, Hou-Tong, Roland Kersting, and Gyu Cheon Cho. "Terahertz imaging with nanometer resolution." Applied Physics Letters 83.15 (2003): 3009-3011.

[13] Brun, Marc-Aurèle, et al. "Terahertz imaging applied to cancer diagnosis." Physics in Medicine \& Biology 55.16 (2010): 4615.

[14] Mittleman, Daniel. "Terahertz imaging." Sensing with terahertz radiation. Springer, Berlin, Heidelberg, 2003. 117-153.

[15] Tonouchi, Masayoshi. "Cutting-edge terahertz technology." Nature photonics 1.2 (2007): 97-105.

[16] Zangeneh-Nejad, Farzad, and Reza Safian. "Significant enhancement in the efficiency of photoconductive antennas using a hybrid graphene molybdenum disulphide structure." Journal of Nanophotonics 10.3 (2016): 036005.

[17] Jiang, Zhiping, and Xi-Cheng Zhang. "Terahertz imaging via electrooptic effect." IEEE Transactions on microwave theory and techniques 47.12 (1999): 2644-2650.

[18] Pickwell, E., et al. "Simulation of terahertz pulse propagation in biological systems." Applied Physics Letters 84.12 (2004): 2190-2192.

[19] Gallot, Guilhem, and D. Grischkowsky. "Electro-optic detection of terahertz radiation." JOSA B 16.8 (1999): 1204-1212.

[20] Fukunaga, Kaori, and Marcello Picollo. "Terahertz spectroscopy applied to the analysis of artists' materials." Applied Physics A 100.3 (2010): 591-597.

[21] Zangeneh-Nejad, Farzad, and Reza Safian. "Hybrid graphenemolybdenum disulphide based ring resonator for label-free sensing." Optics Communications 371 (2016): 9-14.

[22] Bolivar, P. Haring, et al. "Label-free probing of genes by time-domain terahertz sensing." Physics in Medicine \& Biology 47.21 (2002): 3815.

[23] Nagel, M., et al. "Integrated THz technology for label-free genetic diagnostics." Applied Physics Letters 80.1 (2002): 154-156.

[24] Yang, Yuping, Dongqian Xu, and Weili Zhang. "High-sensitivity and label-free identification of a transgenic genome using a terahertz metabiosensor." Optics express 26.24 (2018): 31589-31598.

[25] Zangeneh-Nejad, Farzad, and Reza Safian. "A graphene-based THz ring resonator for label-free sensing." IEEE Sensors Journal 16.11 (2016): 4338-4344.

[26] Bai, Jingwei, et al. "Graphene nanomesh." Nature nanotechnology 5.3 (2010): 190-194.

[27] Bai, Jingwei, et al. "Graphene nanomesh." Nature nanotechnology 5.3 (2010): 190-194.

[28] Mikhailov, Sergey A., and Klaus Ziegler. "New electromagnetic mode in graphene." Physical review letters 99.1 (2007): 016803.

[29] Avouris, Phaedon, and Christos Dimitrakopoulos. "Graphene: synthesis and applications." Materials today 15.3 (2012): 86-97.

[30] Avouris, Phaedon, and Christos Dimitrakopoulos. "Graphene: synthesis and applications." Materials today 15.3 (2012): 86-97.

[31] Zangeneh-Nejad, Farzad, and Amin Khavasi. "Spatial integration by a dielectric slab and its planar graphene-based counterpart." Optics letters 42.10 (2017): 1954-1957.

[32] Gao, Wei. "The chemistry of graphene oxide." Graphene oxide (2015): 61-95.

[33] Bhuyan, Md Sajibul Alam, et al. "Synthesis of graphene." International Nano Letters 6.2 (2016): 65-83.

[34] Andrew, Richard Charles, et al. "Mechanical properties of graphene and boronitrene." Physical review B 85.12 (2012): 125428.

[35] Bolivar, P. Haring, et al. "Label-free probing of genes by time-domain terahertz sensing." Physics in Medicine \& Biology 47.21 (2002): 3815.

[36] Yoo, Byung Min, et al. "Graphene and graphene oxide and their uses in barrier polymers." Journal of Applied Polymer Science 131.1 (2014).

[37] Choi, Wonbong, and Jo-won Lee, eds. Graphene: synthesis and applications. CRC press, 2011.

[38] Bacon, Mitchell, Siobhan J. Bradley, and Thomas Nann. "Graphene quantum dots." Particle \& Particle Systems Characterization 31.4 (2014): 415-428.

[39] Peplow, Mark. "Graphene: the quest for supercarbon." Nature News 503.7476 (2013): 327. 
bioRxiv preprint doi: https://doi.org/10.1101/2021.03.07.434261; this version posted March 8,2021 . The copyright holder for this preprint (which was not certified by peer review) is the author/funder. All rights reserved. No reuse allowed without permission.

> REPLACE THIS LINE WITH YOUR PAPER IDENTIFICATION NUMBER (DOUBLE-CLICK HERE TO EDIT) <

[40] Wu, Yanqing, et al. "State-of-the-art graphene high-frequency electronics." Nano letters 12.6 (2012): 3062-3067.

[41] Zangeneh-Nejad, F., \& Safian, R. (2015, May). A tunable highimpedance $\mathrm{THz}$ antenna array. In 2015 23rd Iranian Conference on Electrical Engineering (pp. 402-405). IEEE.

[42] Kim, Jaemyung, et al. "Graphene oxide sheets at interfaces." Journal of the American Chemical Society 132.23 (2010): 8180-8186.

[43] Zbořil, Radek, et al. "Graphene fluoride: a stable stoichiometric graphene derivative and its chemical conversion to graphene." small 6.24 (2010): 2885-2891

[44] Beard, Matthew C., Gordon M. Turner, and Charles A. Schmuttenmaer. "Terahertz spectroscopy." (2002): 7146-7159.

[45] Ambrosi, Adriano, et al. "Electrochemistry of graphene and related materials." Chemical reviews 114.14 (2014): 7150-7188.

[46] Brun, Marc-Aurèle, et al. "Terahertz imaging applied to cancer diagnosis." Physics in Medicine \& Biology 55.16 (2010): 4615.

[47] Zheng, Gaige, et al. "High-resolution surface plasmon resonance sensor with Fano resonance in waveguide-coupled multilayer structures." Applied Physics Express 10.4 (2017): 042202.

[48] Zangeneh-Nejad, Farzad, and Romain Fleury. "Topological fano resonances." Physical review letters 122.1 (2019): 014301.

[49] De Guevara, ML Ladron, F. Claro, and Pedro A. Orellana. "Ghost Fano resonance in a double quantum dot molecule attached to leads." Physical Review B 67.19 (2003): 195335.

[50] Babicheva, Viktoriia E., and Andrey B. Evlyukhin. "Resonant lattice Kerker effect in metasurfaces with electric and magnetic optical responses." Laser \& Photonics Reviews 11.6 (2017): 1700132. 